\title{
Human resource allocation to multiple projects based on members' expertise, group heterogeneity and social cohesion
}

Article

Accepted Version

Ballesteros-Pérez, P., Phua, F. T. T. and Mora-Melià, D. (2019) Human resource allocation to multiple projects based on members' expertise, group heterogeneity and social cohesion. Journal of Construction Engineering and Management, 145 (2). ISSN 0733-9364 doi: https://doi.org/10.1061/ (ASCE)CO.1943-7862.0001612 Available at https://centaur.reading.ac.uk/78762/

It is advisable to refer to the publisher's version if you intend to cite from the work. See Guidance on citing.

To link to this article DOI: http://dx.doi.org/10.1061/(ASCE)CO.19437862.0001612

Publisher: American Society of Civil Engineers

All outputs in CentAUR are protected by Intellectual Property Rights law, including copyright law. Copyright and IPR is retained by the creators or other copyright holders. Terms and conditions for use of this material are defined in the End User Agreement. 


\section{CentAUR}

Central Archive at the University of Reading

Reading's research outputs online 
3 Pablo Ballesteros-Pérez, Ph.D. ${ }^{*}$; Florence Ting Ting Phua, Ph.D. ${ }^{2}$; Daniel Mora-Melià, Ph.D. ${ }^{3}$

\section{Abstract}

5 Project managers regularly allocate human resources to construction projects. This critical 6 task is usually executed by fulfilling the minimum project staffing requirements normally

7 based around the quantity and competence of project members. However, research has shown

8 that team performance can increase by up to $10 \%$ and $18 \%$, respectively, as a consequence of

9 the group members' heterogeneity and social cohesion. Also, there is currently no practical

10 quantitative tool which incorporates these aspects to allow project managers to achieve this

11 task efficiently and objectively.

12 A new quantitative model for the effective allocation of human resources to multiple projects, which takes into account group heterogeneity and social cohesion is proposed. This model is easy to build, update and use in real project environments with the use of a spreadsheet and a basic optimization engine (e.g. Excel Solver). A case study is proposed and solved with a Genetic Algorithm to illustrate the model implementation. Finally, a validation example is

17 provided to exemplify how group heterogeneity and social cohesion condition academic achievement in an academic setting.

\footnotetext{
${ }^{1}$ Senior Lecturer. School of Architecture, Building and Civil Eng., Loughborough University, Loughborough, Leicestershire LE11 3TU, UK. *Corresponding author: +44 (0) 150922 3771, p.ballesteros-perez@lboro.ac.uk 2 Associate Professor. School of the Built Environment. University of Reading, Whiteknights, Reading RG6 6DF, UK. Phone: +44 (0) 118378 8194, f.phua@reading.ac.uk

${ }^{3}$ Associate Professor. Departamento de Ingeniería y Gestión de la Construcción, Universidad de Talca, Facultad de Ingeniería, Curicó, camino Los Niches km.1 3340000, Chile. Phone: +56 951265 044, damora@utalca.cl
} 
20 Keywords

21 Human resource management; human resource allocation; team performance; heterogeneity;

22 faultlines; cohesion; sociometry

\section{Introduction}

It is a well-known saying that 'people are the lifeblood of organizations'. Indeed, despite living in an era of constant technological advancement, most of our tasks are still done, handled or supervised by human beings. In organization life, the size and/or complexity of many undertakings nowadays demand the involvement of many people (sometimes from different organizations) working together to achieve a common goal. This goal can be anything, but many times involves creating deliverables (products, services) to enhance a company's internal performance, to make profit, or both. However, people (employees, workers) who take part in these undertakings are normally subject to constraints. For example, they are qualified to do certain jobs and not others; they have different levels of competence in different domains; they cannot be present in multiple locations; and, certainly, they have physical constraints in terms of how long they can work for (Hendriks et al. 1999). Therefore, when there are several, sometimes concurrent projects that require the participation of people to be completed, a project manager faces a practical dilemma: how to best allocate his/her human resources on-hand to deliver his/her projects successfully. 'Successfully' can mean completing the projects on time, on budget and within an agreed (or shared) quality threshold, or just meeting the key stakeholders' expectations (Xia et al. 2017).

41 In any case, as long as there are ongoing projects, the project manager will require competent human resources to engage in certain tasks for a period of time before they are freed and able 
to join other ongoing or upcoming projects. An essential part of the project manager's role in the allocation of optimum human resources is to ensure, as much as possible, that the individuals within the projects can work cooperatively with each other (Anvuur and Kumaraswamy 2016).

However, collaboration between project members does not happen by chance. There are indeed many factors that prevent this from happening. These factors can be communication-related for instance, and/or have to do with the project member's demographic attributes such as (differences in) nationality, education, religion, experience, to cite a few (Al-Bayati et al. 2017). Sometimes, there are people who do not like working with certain individuals, and this can also be really detrimental to the project progress and its eventual success (Chen et al. 2017b). In this regard, Phua (2004) and Phua and Rowlinson (2004) have found that cooperative behavior between project members is influenced, to a certain extent by individual members' intrinsic social and psychological factors which have to do with many more factors other than just their extrinsic demographic profile such as age, sex, education, work experience and roles. For this reason, we will consider both cohesion and heterogeneity factors later when aiming to build high-performing teams.

Given our existing understanding of the various factors that affect team performance, there is however, a scarcity of quantitative and objective tools that enable the effective allocation of human resources in terms of where and when they are to be allocated to projects (Ahmadian Fard Fini et al. 2017). Conventionally, this type of allocation issues largely fall within mainstream Human Resource Management (HRM) application which has its roots in social sciences.

A different, maybe opposite, scenario can be found within Operational Research (OR), which deals with the modeling and application of advanced analytical methods to make better decisions. The problem of allocating multiple human resources to a single project is 
relatively recent in OR, but it has been well studied and is known nowadays as the 'Team Formation Problem' (TFP) (Tseng et al. 2004). When there are multiple simultaneous projects, the TFP becomes the 'Multiple Team Formation Problem' (MTFP). Particularly, the grouping of individuals to create teams have been made by attending to multiple factors: the resources' temporal availability, current workload, individuals' skills, level of competence, geographical distance, seniority, number of contacts, among many others (Gutiérrez et al., 2016). In this line of research, it is not common to find theoretically-grounded sociological considerations in the composition of teams. This means that, whereas it is relatively easy to come across OR models that allocate resources that meet some functional (e.g. skills, competence) project members' requirements, it is very rare to find models that try to optimize other socially-based group traits like intra-group social preferences and group cohesion (Ballesteros-Pérez et al. 2012). This piece of research proposes to take a step forward in bridging this gap.

In this paper, a new human resource allocation model that takes into account, not just basic project staff requirements and employees' profiles, but also group heterogeneity (diversity) and social cohesion, is developed. This is a worthwhile contribution because, as discussed earlier, team performance has been demonstrated to be significantly influenced by these two factors. Hence, it seems logical to incorporate this knowledge when creating highfunctioning teams which comprise the 'right' individuals working together. To this end, the rest of the paper will be structured as follows. The literature review section will go over the major contributions published in the areas of the MTFP, group heterogeneity and social cohesion. The materials and methods section will formulate the model, define its major variables and explain how these are interrelated under mathematical expressions for measuring team performance. A case study will exemplify the model implementation in a fictitious company environment with twenty people and three simultaneous projects. A short 
validation section will implement the model in a real academic setting where a cohort of 15 MSc students worked in groups to deliver three projects. The discussions will provide some insight and further analysis on the implications and limitations of the model. Finally, the conclusions will summarize the paper and convey why the proposed tool is relevant to the wider project management community.

\section{Literature review}

The proposed model draws from research developed in two very different areas operational research $(\mathrm{OR})$ and applied psychology $(\mathrm{AP})-$, but it is applied on a third one: Human Resource Management (HRM). The amount of works published in connection with HRM within both OR and AP is endless, so it is necessary to narrow down significantly the works to be presented here. In this regard, only three very relevant topics will be reviewed: the MTFP, group heterogeneity and faultlines, and group cohesion and sociometry.

\section{The Multiple Team Formation Problem (MTFP)}

The MTFP involves the distribution of people with different skillsets to a series of teams (projects) that usually require more than a single area of expertise while optimizing other criteria (e.g. profits, execution time, number of people). This problem is known to be NP-hard (Non-deterministic Polynomial-time Hard) even for instances with a single project (Gutiérrez et al., 2016). This means the MTFP belongs to the set of OR problems that are harder to solve.

The first attempt to model and compute a solution to the TFP is relatively recent and was developed by Lappas et al. (2009) when trying to create teams of experts from professional profiles posted on social networks. Just a year later, Dorn and Dustdar (2010) 
117 proposed solving the TFP with a first heuristic approach, whereas Li and Shan (2010)

118 improved the Enhanced-Steiner algorithm that was one of the two original algorithms used to 119 solve the TFP.

A year later, Yin et al., (2011) were the first to consider social influence among the

121 teams of experts. Additionally, Farhadi et al. (2011) allowed for the possibility of different

122 competence levels among the human resources, a generalization that will also be considered 123 in our model.

In 2012, the number of works published on the TFP grew exponentially. Among the most relevant: Sorkhi et al. (2012) proposed a game theoretic approach to form and rank project teams; Farhadi et al. (2012a, 2012b) extended the second original algorithm that had

127 proven to be very effective when dealing with the TFP - the Rarest First algorithm -; whereas Gajewar and Sarma (2012) proposed three new optimization algorithms and successfully applied them to the MTFP for the very first time.

Next, Shi and Hao (2013) formulated the MTFP with a multi-criteria decision-making ranking approach involving the individuals' social networks. Then, Teixeira and Huzita

132 (2014) approached the MTFP considering the human resources' contextual information

133 (culture, idiom, temporal distance and previous experience), besides task requirements and the interpersonal relationships among human resources. Our proposed model will also take advantage of similar constructs in order to create a multi-dimensional model. Also, Agrawal et al. (2014) focused on educational settings allowing the MTFP to be implemented without

137 allowing overlaps between the different student teams, a feature that will also be considered 138 in our model. Still in the same year, Awal and Bharadwaj (2014) tried to capture the synergy

139 produced among team members by means of a new ad-hoc concept named 'Collective

140 Intelligence' and also used a Genetic algorithm to solve their problem formulation. In this

141 paper, the solution of the case study proposed later will also make use of a genetic algorithm 
142 approach as the way this ad-hoc index was defined share some similarities with our objective 143 function.

144 Although there have been many other recent works published on the MTFP, these will 145 not be recounted here as they are not directly germane to this study. However, one that is

146 perhaps worth highlighting is the work from Gutiérrez et al. (2016) which formally included

147 sociometric preferences among individuals in the MTFP. Our proposed model also shares a 148 similar approach for modeling group cohesion. However, the algorithmic approach will be 149 totally different to Gutiérrez et al.'s as our model includes other dimensions, which makes 150 our model no longer quadratic.

\section{Group heterogeneity and faultlines}

Research on how team effectiveness is influenced by the team composition has been abundant too. Most of this research has focused precisely on measuring and analyzing the effects of group heterogeneity on team performance. Group heterogeneity (homogeneity) refers to a measurement of how different (similar) the members' demographic attributes (age, sex, ethnicity, etc.) are with each other. There are many reviews on group heterogeneity (see

158 Earley and Gibson (2002) for a comprehensive one) but they will not be recounted here 159 either. In this piece of research, we are focusing on the quantitative aspects of how 160 heterogeneity is measured and what are its effects on team performance, rather than the 161 mechanisms or factors that cause it.

163 (homogeneous/heterogeneous) a group can be were defined by Blau (1977) and Allison

164 (1978). Generally, these and other later indices involved measuring group homogeneity as the 
members' demographic attribute overlaps. With those indices, heterogeneity was also generally defined as the inverse of homogeneity, that is heterogeneity $=1 /$ homogeneity.

Additionally, for a long time, it was believed that the presence of faultlines (demographic features that divide a bigger group into two or more relatively homogeneous subgroups) was detrimental to group performance (Lau and Murnighan 2005). It was not until the work of Gibson and Vermeulen (2003), who proposed a new metric for measuring group heterogeneity - the Subgroup Strength - , that it was understood that the presence of subgroups (faultlines) could indeed promote team learning behavior and improve their performance. The Subgroup Strength (SS) has many advantages over previous homogeneity metrics (indices) as it allowed researchers to identify group faultlines much more effectively. Indeed, it was shown recently by Meyer and Glenz (2013) in a comprehensive comparative study that the SS is one of the simpler, yet more powerful metrics for measuring group heterogeneity in the presence of two or more subgroups. For these reasons, SS will also be used in our model later to describe subgroups' heterogeneity.

Finally, Gibson and Vermeulen (2003) also showed that a team's performance seemed to vary by up to $10 \%$ depending on the SS. Again, this was supported recently by another study by Chen et al. (2017). This study also confirmed another speculation of Gibson and Vermeulen's: that the relationship between SS and team performance was an inverted Ushape whose minima (lower performance) were to be expected for extremely homogeneous and heterogeneous groups. Finally, many other works have been published on the effects of group heterogeneity on intra- and cross-subgroups demographic faultlines (Lau and Murnighan 2005), but only some related to group cohesion will be reviewed later in the Discussions to clarify the effect of possible collinearities between both variables. 
Group cohesion is a desirable attribute because research has proven it to be positively related to team performance, as well as a wide range of other positive behavioral outcomes (better individuals' attitude, well-being, lower absenteeism, etc.) (Chang and Bordia 2001; Chen et al. 2017b). However, very few pieces of research have actually quantified the extent to which team performance is influenced by group cohesion or dissociation. percentile points on average above the average (uncohesive) groups. This figure will be used later in our model as other research has also corroborated the cohesion-performance relationship even when different settings (e.g. business, education, research) or group sizes are considered (Castaño et al. 2013). Furthermore, because existing research on cohesion and performance has operationalized cohesion almost completely in terms of interpersonal attraction (see evidence from Lott and Lott (1965) to Beal et al. (2003) for instance), it makes theoretical sense for our model to adopt sociometry to model group cohesion. can be used for estimating the quality of group dynamics. It is one of the few methods that allows the gathering of quantitative information about the informal structure of a group that is difficult to obtain in other ways. Sociometry was extensively used between the 40 s and 60 s at schools, companies and research settings to examine social interrelations and communication 210 patterns within groups (Salo 2006). In sociometry, interpersonal relations are measured by asking group members to express their preferences and rejections for particular companions in a certain situation or activity (Festinger et al. 1950). Hence, the advantageous simplicity of sociometry is, at the same time, its major limitation: it requires that group members are 
214 truthful and open in stating who they prefer and not prefer to work with. A reasonable

215 question then is whether group cohesion can be adequately represented by sociometric

216 choices and if these choices $r$ can be eventually captured by means of questionnaires that

217 request group members to state their preferences and rejections towards other group

218 members. In fact, both aspects have been subjected to multiple research studies in many

219 varied settings. An example of a brief but reassuring and confirmatory review can be found in

220 Salo (2006).

Finally, there is one question that needs to be addressed before formulating the model.

222 As stated earlier, the proposed model will group individuals under different projects that have

223 some minimum staff (areas of expertise and levels of competence) requirements. According

224 to a recent piece of research (Mathieu et al. 2015), when people with the right combination of expertise work together, as expected, this is positively related with team performance.

However, this same piece of research also showed that this is unrelated to team cohesion.

227 With this in mind, we will allow our model to effectively separate the effect of the constraints

228 (i.e. minimum project staffing requirements) from the group performance variables (i.e.

229 group heterogeneity and cohesion metrics).

\section{Materials and methods}

\section{Model outline}

In this section, an OR model that allocates a pool of skilled individuals to a series of

234 simultaneous projects with specific staffing (expertise and competence) requirements is

235 proposed and mathematically described in detail. This model will take into account how

236 similar (homogeneous) these individuals are and how they get along with each other (group cohesion). 


\section{Mathematical notation}

240

Let us assume two individuals $i$ and $j$ where $i, j$ belong to a set of $n$ people (workers)

241 who are available to be allocated into teams. Let us assume that these individuals can be

242 combined into a number of non-overlapping teams (subgroups) where each team is noted by

243 the letter $k$ and whose size is noted as $n_{k}$ (number of members of team $k$ ).

$244 \quad$ For every individual $i$ (or $j$ ) it is assumed that the following information is known as 245 illustrated in the following examples:

246 - Professional level of competence $l_{i}$ where $l_{i} \in L$ and $L=\{$ junior, intermediate, senior $\}$

247 - Functional department $d_{\text {i }}$ where $d_{i} \in D$ and $D=\{$ architecture, civil, mechanical, electrical $\}$

$248 \quad$ Age $a_{i}$ where $a_{i}=$ positive integer.

$249-$ Gender $g_{\mathrm{i}}$ where $g_{i} \in G$ and $G=\{$ Male, Female $\}$

250 Ethnicity $e_{\mathrm{i}}$ where $e_{i} \in E$, and where $E$, for simplicity, will be assumed here as the

251 continent of origin, that is $E=\{$ African, Antarctican, Asian, Australian, European, North

252 American, South American $\}$

253

- Team tenure (seniority in the same group or company) $t_{i}$ where $t_{i}=$ positive integer.

254

- $\quad$ Sociometric preference of individual $i$ towards individual $j$, that is $s_{i j}$ where $i \neq j, s_{i j} \in S$ and $S=\{-1,0,+1\}$. Particularly, $s_{i j}=-1$ means $i$ dislikes working with $j, s_{i j}=0$ means $i$ is neutral towards (or has never worked with) $j$, and $s_{i j}=+1$ means $i$ likes working with $j$. The set of all values $s_{i j}$ correspond to a non-symmetrical matrix of size $n \times n$.

Sociometric preferences aside, these individuals' attributes have been selected here as they were the ones adopted by Gibson and Vermeulen (2003) in their seminal work on group faultlines. This set of attributes has been widely tested in subsequent research (e.g. Chen et 
al. 2017a; Meyer and Glenz 2013) and it is still largely accepted that they provide a robust

262

263

264

265

266

267

268

269

270

271

272

273

274

275

276

277

278

279

280

281

282

283

284 representation of group diversity.

Hence, given $n$ people available from whom we know their $l_{i}, d_{i}, a_{i}, g_{i}, e_{i}, t_{i}$ and $s_{i j}$, we will create subsets (subgroups/teams) of $n_{k}$ individuals, each of which will be working on a different project $k$. Individuals can only be allocated to either a single group $k$ or no subgroup at all (those unallocated individuals will be idle resources). This implies that no individual can be present in two or more subgroups, even if they could only work part-time in several projects. We use this simplified assumption to make this model more accessible from the point of view of its first mathematical formulation.

Therefore, as implied above, every subgroup $k$ will be allocated to a single project and we will note projects and subgroups (teams) with the same subscript $k$ from now on. Each project $k$ will have specific staffing requirements $\left(p_{k}\right)$. For instance, $p_{k}=\{1$ senior Architect, 1 intermediate civil engineer, 1 junior civil engineer, 2 intermediate electrical engineers $\}$. Any subgroup of workers $n_{k}$ that matches or exceeds (both in number and/or competence) these requirements will be considered a feasible subgroup that can potentially be allocated to project $k$.

\section{Team performance measurement}

In order to determine which feasible allocation of subgroups is most desirable, it is necessary to anticipate how much better each possible alternative allocation of subgroups would perform if eventually chosen. Additionally, it is worth emphasizing that each feasible allocation might encompass multiple subgroups as each subgroup will be allocated to one project. Therefore, it is necessary to create an index that captures, not just how efficient each subgroup is, but also how efficient all groups are on average; that is, how efficient the 
allocation is altogether. This index will be named 'Global Efficiency (E)' and will correspond

to a weighted average calculated from the subgroup Efficiencies of each subgroup $k$ (noted as

$\left.287 E_{k}\right)$, that is:

$$
E=\sum E_{k} \cdot w_{k}
$$

In this expression, $w_{k}$ corresponds to the weight of each subgroup $k$. This way $w_{k}$ can be calculated, for instance, proportionally to each project $k$ 's budget $\left(b_{k}\right)$. Alternatively, $w_{k}$ can also be calculated proportionally to the number of people $n_{k}$ from each project, divided by the total people available $n$ (allocated or not) or the total number of allocated people only

$\left(\sum w_{k}\right)$. These alternatives are expressed in equations (2) and (3), respectively:

$$
w_{k}=\frac{b_{k}}{\sum b_{k}}
$$

$$
w_{k}=\frac{n_{k}}{\sum n_{k}} \quad \text { or } \quad w_{k}=\frac{n_{k}}{n}
$$
subgroup's $E_{k}$ and $w_{k}$ values, now it is necessary to detail how $E_{k}$ values can be calculated. $E_{k}$ is a composite efficiency index obtained as the product of two other indices that represent the expected performance of that subgroup $k$ in terms of its homogeneity $\left(P_{k}^{S S}\right)$ and social cohesion $\left(P_{k}^{S}\right)$. Namely,

$$
E_{k}=P_{k}^{S S} \cdot P_{k}^{S}
$$

Strength (SS) as defined by Gibson and Vermeulen (2003). To calculate the SS value of a subgroup $k$ (noted as $S S_{k}$ ), it will be necessary to calculate the subgroup $k$ 's homogeneity value $h_{k}$ first, as well as the individuals' degree of overlaps in terms of different diversity 
factors (we will use: functional department, age, gender, ethnicity, and team tenure as

307 justified later).

subgroup $k$ given a particular level of cohesion, which is measured by sociometric indices. In

310 this case, $S_{k}$ will be calculated as the interpersonal social preferences and rejections stated by

311 all members belonging to subgroup $k$.

$$
h_{k}=\frac{\sum_{i<j}\left\{O_{i j}^{d}+O_{i j}^{a}+O_{i j}^{g}+O_{i j}^{e}+O_{i j}^{t}\right\}}{\frac{n_{k}\left(n_{k}-1\right)}{2}}=\frac{\sum_{i<j} O_{i j}}{\frac{n_{k}\left(n_{k}-1\right)}{2}}
$$

Where $O_{i j}$ is the total overlap between individuals $i$ and $j$, and which is computed as

318 the sum of $O_{i j}^{d}, O_{i j}^{a}, O_{i j}^{g}, O_{i j}^{e}$ and $O_{i j}^{t}$ which, in turn, represent the overlaps between two

319 individuals $i$ and $j$ on functional department, age, gender, ethnicity and team tenure,

320 respectively. The sum in the numerator is restrained to $i<j$ (but it could have also been $i>j$ indistinctly) to avoid the cases where $i=j$ (individuals' self-overlaps) as well as to prevent the symmetrical $O_{i j}$ values (that is $O_{i j}=O_{j i}$ ) from being counted twice. where $i \geq j)$. 

overlaps between a group of individuals can be calculated as follows:

329

Age overlap:

$$
O_{i j}^{a}=\frac{\min \left(a_{i}, a_{j}\right)-18}{\max \left(a_{i}, a_{j}\right)-18}
$$

$$
O_{i j}^{g}=1 \text { if } g_{i}=g_{j} \text {, else } 0
$$

$$
O_{i j}^{e}=1 \text { if } e_{i}=e_{j} \text {, else } 0
$$

$$
\text { Team tenure overlap: } \quad O_{i j}^{t}=\frac{\min \left(t_{i}, t_{j}\right)}{\max \left(t_{i}, t_{j}\right)}
$$
Overlap values can vary between $[0,1]$. Hence, values of $h_{k}$ will vary between $[0,5]$.

334 We are aware that other diversity factors could have also been included in the definition of $h_{k}$ 335 such as for example, language, education, experience. However, in the interest of keeping to 336 the model's simplicity and for illustrative purpose in the case study which follows, we 337 deemed it reasonable to stick to the diversity factors in the definition of $h_{k}$ as proposed by 338 Blau (1977) and Allison (1978). And now that the overlaps of all individuals $O_{i j}$ and the subgroup $k$ 's homogeneity 340 value $h_{k}$ have been detailed, the Subgroup Strength of a subgroup $k\left(S S_{k}\right)$ is defined as the 341 population standard deviation of the $O_{i j}$ values from all $n_{k}$ members belonging to subgroup $k$, 342 that is:

$$
S S_{k}=\sqrt{\frac{\sum_{i<j}\left(O_{i j}-h_{k}\right)^{2}}{\frac{n_{k}\left(n_{k}-1\right)}{2}}}=\text { Std. Dev. } O_{i j}
$$


345 Additionally, Gibson and Vermeulen (2003) proved that team diversity (represented by

346 means of $S S_{k}$ ) and group performance were quadratically related (inverted U-shape)

347 approximately as described in Figure 1a.

Also, a recent study by Chen et al. (2017) suggested that this quadratic expression is quasi-symmetrical and that the value of $\delta$ seems generally close to $10 \%$ on average.

351 Therefore, the subgroup k's performance $P_{k}^{S S}$ can be calculated from the subgroup strength $S S_{k}$ value as:

$$
P_{k}^{S S}=-2.56 \delta S S_{k}^{2}+3.2 \delta S S_{k}+1-\delta \quad \text { with } \delta \approx 0.10
$$
calculated from the subgroup $k$ members' sociometric preferences $s_{i j}$ towards each other (i.e. preferences and rejections to work with a particular individual). These preferences and rejections do not have to be symmetrical (that is, $S_{i j} \neq S_{j i}$ or $S_{i j}=S_{j i}$ ). Hence, we define a subgroup $k$ 's cohesion $S_{k}$ as:

$$
S_{k}=\frac{\sum_{i \neq j} s_{i j}}{n_{k}\left(n_{k}-1\right)}
$$
excluding the choices of individuals with themselves. So, as $s_{i j}$ can be equal to -1 (meaning $i$ dislikes $j$ ), 0 ( $i$ is neutral or have not met $j$ ), or +1 ( $i$ likes $j), S_{k}$ actually represents how well 
365 (or badly) all subgroup $k$ 's members get along with each other on average. Analogously, $S_{k}$ can take on values within the range $[-1,1]$.

367 Finally, previous researchers' results suggest that the average cohesive group seems to perform around $18 \%$ better than average (non-cohesive or non-uncohesive) groups (Evans and Dion 2012). For the purpose of this paper, this performance differential will be called $\varphi$. However, it is worth pointing out that in those previous pieces of research it is not always

371 clear how group cohesion is measured or quantified. Also, there is a total absence of studies

372 clarifying whether the cohesion-performance relationship is linear or if it indeed follows a

373 different pattern. In light of this, it seems prudent to take the simplest alternative and assume

374 that group cohesion (represented now by $S_{k}$ ) and performance $\left(P_{k}^{S}\right)$ will just be linearly

375 related as represented in Figure 1b. Hence:

$$
P_{k}^{S}=1+\varphi S_{k} \quad \text { with } \varphi \approx 0.18
$$
to each other. We are now able to calculate the global group efficiency (E) from the different 379 simultaneous subgroups' efficiencies $E_{k}$ and their respective weights $w_{k}$. This is summarized 380 at the bottom of Figure 1. Hence, from now on, every possible subgroups' allocation can be measured in relative performance terms and each feasible complete group allocation can be compared against each other. The following is an example to illustrate how we can apply the model based on a fictitious case study which reflects as much as possible, a real project environment. 


\section{Application}

387

388

389

390

391

392

393

394

395

396

397

398

399

400

401

402

403

404

405

406

407

408

409

The case study follows approximately the same order of calculations that was presented in the previous section. For the interested reader, the complete step-by-step calculations can be found in an Excel file accessible from the Supplemental Online Material.

Particularly, this case study comprises a group of 20 individuals with different levels of professional competence and who belong to four functional departments. The entire professional, demographic (homogeneity-related) and sociometric (cohesion-related) information from the 20 individuals is described in Figure 2.

\section{<Insert Figure 2 here $>$}

These 20 individuals are to be allocated to three simultaneous projects, each of which has different staffing (professional level and functional department) requirements as well as budgets. This information is detailed in Figure 3. Also, at the bottom of Figure 3, the weights of each project have been calculated as a function of the project budgets according to expression (2).

\section{$<$ Insert Figure 3 here>}

Any subgroup allocation that meets or exceeds the staffing requirements described in Figure 3 will be a feasible solution. What is necessary now is to be able to calculate the global efficiency E from any feasible grouping solution. With this aim in mind, the first step will be to obtain all individuals' overlaps concerning functional department, age, gender, ethnicity and team tenure, so that these values can be reused anytime when two individuals are put together in the same subgroup. Due to its length (5 tables, one per type of overlap), these calculations have been included as Supplemental Online Material. Figure 4 just summarizes the total overlaps, which have been obtained from the simple addition of all the individuals' overlap values. 
411 What remains is calculating, for any potential and feasible subgroup $k$, its

412 homogeneity $h_{k}$ (with expression (5)), subgroup strength $S S_{k}$ (with expression (11)), and

413 cohesion $S_{k}$ (with expression (13)) values. Then, with $S S_{k}$ and $S_{k}$, known, calculating the

414 homogeneity-related $P_{k}^{S S}$ performance metric (with expression (12)) and the cohesion-related

$415 P_{k}^{S}$ performance metric (with expression (14)) can be performed. Next, with $P_{k}^{S S}$ and $P_{k}^{S}$

416 known, we can calculate $E_{k}$ (by means of expression (4)). Once the values of $E_{k}$ are all known

417 for all the simultaneous subgroups (three in our example, as there are three projects), and by

418 knowing the weight of each subgroup $w_{k}$ (with expressions (2) or (3), and as detailed at the

419 bottom of Figure 3), it is possible to obtain the global efficiency E of that group configuration

420 by means of expression (1). This series of calculations are represented vertically from top to

421 bottom in the lower half of Figure 5.

\section{$<$ Insert Figure 5 here $>$}

423

In Figure 5, a random solution directly allocating the individuals available to meet the project staffing requirement, but without any further (homogeneity, nor cohesion) considerations, is presented. At the top of Figure 5, one can find the allocation of each individual to each subgroup/project. The column to the right sums each individual's

427 allocations and verifies that no individual is allocated more than $100 \%$, that is, to more than one project. These are necessary but not sufficient problem constraints which need to be met to qualify any allocation as feasible.

However, every time there is any change (for instance a member is allocated to a

431 different project/subgroup), all values need to be recalculated. Therefore, the only way of

432 finding good solutions is by iterating these calculations multiple times while testing as many 
433 feasible solutions as possible. Hence, for the model to be practically useful, this task must be

434

435

436

437

438

439

440

441

442

443

444

445

446

447

448

449

450

451

452

453

454

455

456 automated by an optimization algorithm.

The global efficiency of the random solution depicted in Figure 5 corresponds to

0.981. By definition, the value of the global efficiency E will vary between $[1-\delta-\varphi, 1+\varphi]$.

Hence, the closer the value of $\mathrm{E}$ to $1+\varphi$, the higher the expected groups' performance. Now, in looking for that optimum solution, there is one last point to be discussed.

As discussed earlier, the proposed model falls within a particular case of the MTFP.

The MTFP is NP-hard, and that opens the door to the use of metaheuristics when looking for (near) optimum solutions. Among common metaheuristics, Genetic Algorithms (GA) are one of the quicker and simpler, but also effective, options. On top of that, they are usually available within commercial solvers included by default in spreadsheet software like Microsoft Excel ${ }^{\circledR}$. Moreover, GA have been in use in resource-constrained allocation for a long time and they have been considered as one of the most efficient metaheuristics when dealing with the MTFP in recent studies (e.g. Ahmadian Fard Fini et al. 2016; Awal and Bharadwaj 2014). For all these reasons, we will use GA for solving multiple instances of our case study and find a quick and good (despite maybe less than optimal) solution.

Therefore, once the model is implemented in a spreadsheet where every time a new allocation is proposed all the subgroup calculations can be automatically and instantly updated, the GA can start looking for new solutions. The problem constraints are the ones specified in grey cells (one per individual's maximum allocation time plus one per project staffing requirements check). The objective function corresponds to E, which on this occasion is to be maximized. On resorting to Excel Solver, the best solution was found in seconds by our GA as shown in Figure 6.

\section{$<$ Insert Figure 6 here $>$}



than the one from Figure 5 (with $\mathrm{E}=0.981$ ). If the same GA would have been aimed at minimizing $\mathrm{E}$, the worst solution found (not included) would have had an $\mathrm{E}=0.911$. Within

460 the $[1-\delta-\varphi, 1+\varphi]$ interval, the three solutions correspond to the following percentiles: $56.7 \%$ 461 (the random solution), $79.8 \%$ (the best solution) and $41.5 \%$ (the worst solution). As can be seen, based on the results, there seems to be valid reasons to try to optimize the model outputs with the help of an optimization algorithm. Mostly, when doing this manually, would have been an unsurmountable task.

\section{Validation}

In the previous section it was shown how the model can be implemented to fulfill its most common purpose: finding the optimum (or near optimum) allocation of a set of available human resources into a series of projects, each with not necessarily equal staffing requirements. However, before accepting that the model outputs constitute a fair description

471 of reality, it is necessary to verify whether its parameters actually influence different levels of 472 team performance. Particularly, the most relevant model parameters are the ones proposed in 473 equations (12) and (14), that is, the group diversity-related performance $\left(P_{k}^{S S}\right)$ and the social

474 cohesion-related performance $\left(P_{k}^{S}\right)$. Hence, if higher values of these two parameters exhibit 475 correlation with higher values of team performance, the model will be of some value.

476 Conversely, if there is no such correlation, the model, at least as currently formulated, would 477 render useless.

With this purpose in mind, a first exploratory and validation study was conducted comprising the academic performance of fifteen MSc Civil Engineering students at the Universidad de Talca (Chile). This group of students were enrolled in a module named 
'Projects' which is a transversal integration module and its purpose is to determine how well

482

483

484 students can apply knowledge and understanding from previous related modules. The module was led by one of the authors in the second semester of 2017. It required that fifteen students submitted three assignments (projects) each. The three projects which will be named Project 1, Project 2 and Project 3, had progressive submission dates every two months. Students worked in groups of five to deliver these three projects. After each project was completed, the groups were reshuffled so that most students had to work with different team mates in the next project.

In short, for the first assignment (Project 1), there were three groups with 5 students (named here as groups A, B and C) each submitting a different project. The same happened for Project 2 and 3, but with groups whose member composition was different from Project 1. Each of these three projects was assessed and given a mark between 0 and 100. In total, there were 9 different marks: one per assignment (Project 1, 2 and 3) and group (A, B and C). However, each student only received three marks (one from each Project) whose average resulted in the module's final mark for him/her.

The demographic attributes of the fifteen students can be found in Figure 7. By columns, the five individuals' attributes had a close equivalence with the five attributes described in our model: background (akin to functional department), age, gender, ethnicity and work experience (akin to team tenure). However, as expected from a group of students, the sample was also relatively more homogeneous than other real-life projects (most individuals had similar ages, similar experience, and a less varied set of backgrounds/degrees). 
information. This MSc programme required a minimum work experience of 2 years as an

admission criterion. This is the reason why most students exhibit similar years of experience,

South American. of the same students for a series of lab sessions which ran in parallel to this module.

511 Particularly, before allocating the fifteen students to those lab sessions, they were asked with whom they would like to carry out the lab sessions and whom they would prefer to avoid.

513 Although it was not compulsory for the instructors to implement those preferences, those

514 registers proved useful later for allocating the students to the Project groups, and also to populate the sociometric matrix. student was awarded at the end of the module. correlation analysis between the model parameters and team performance. For this purpose, the groups' homogeneity, subgroup strength and social cohesion values were calculated for the nine five-student groups that submitted the three projects. With these values, calculating the group diversity- and cohesion-related performances was straightforward. These calculations are all presented, along with the students' allocations, in Figure 8.

\section{$<$ Insert Figure 8 here $>$}

Values highlighted in blue, red and green correspond to Projects 1, 2 and 3, which were also identified in the last three columns of Figure 7. For reference purposes, the project marks were also shown at the bottom row of Figure 8 . 

the model align with the expected performance outputs. For this purpose, several plots are shown in Figure 9 from the numerical data represented in the lower rows of Figure 8.

Figures 9a) and 9b) show how subgroup strength and group social cohesion are

533

534

535

536

537

538

539

540

541

542

543

544

545

546

547

548

549

550

551 related to group diversity-related and group cohesion-related performance, respectively, based on equations (12) and (14). Additionally, in Figures 9d) and 9e), we can see how group diversity-related performance and group-cohesion related performance are both related to team performance as well (team performance is represented by the project marks). The very fact that these two graphs exhibit approximately linear trends between the $\mathrm{X}$ and $\mathrm{Y}$ variables indicate that the regression expressions represented in Figures 9a) and 9b) were supported. This is because expressions (12) and (14) are actually transforming the group diversityrelated (from Figure 9a) and group cohesion-related performance (from Figure 9b) into a series of points with a linear correlation with Team Performance. If that had not been the case, then there would be no linear relationship, and quite possibly, no trend would be found at all.

Finally, Figure 9c) represents the Y-axis variables from Figures 9a) and 9b) and barely shows any trend. This proves that the level of correlation between subgroup strength and group cohesion is very low. This relationship was also hypothesized earlier and this is proven numerically and graphically here.

Therefore, a quick observation of the three plots at the bottom of Figure 9 show evidence of a moderate/strong correlation between the model parameters (independent variables) and the project marks (dependent variable). As the project marks can be considered as a good proxy for group performance, we can conclude that the model formulation seems to 
552 be fairly representative and is correctly indicating that certain heterogeneity-related and cohesion-related group attributes can ultimately lead to higher (or lower) team performances. caution too. The analysis is based on an academic environment, rather than a real project.

Real life projects tend to consist of a more diverse group of professionals (higher dispersion of the demographic attributes) with generally many more variables which may be difficult (but necessary) to control. Notwithstanding this, we acknolwedge further validation using real projects is needed in order to improve the validity of the model. However, resorting to an academic environment also has numerous advantages. First, the outcome of 'project'

561 performance can be known (under some simplifying assumptions) as all assignments are

562 graded and awarded a mark. And second, these project cycles are usually faster which also allows data retrieval to be generated faster than in real-life projects. nine) and the reduced variation of some of the performance measurements. In connection with the latter, the cohesion-related performance values of Projects 2 and 3 are very close to each other, obscuring the type of relationship that more dispersed values could have shown.

568 Also, although the rest of the cases show clearer trends, it is necessary to point out that these might not be necessarily linear. This, despite us resorting to three points, two of them still remain too close in the cohesion-related performance graph to infer properly potentially non-

571 liner trends. Finally, it is clear from Figure 9 that Project 1 seemed to be more challenging to the

573 students as they all got lower marks (probably because it was the first assignment), whereas

574 the other two seemed easier (they received higher marks). Similarly, for future validation 575 studies, it will be advisable to gather individual marks from each student (by means of 576 individual exams, for example). Only with this additional piece of information, will it be 
possible to better compare different levels of group members' performance (as groups made up of bright people usually perform better than ones with mediocre students).

579

\section{Discussion}

581

In this paper, a new model for allocating human resources that considers team

582 functional requirements, group heterogeneity and social cohesion has been proposed and

583 validated. In formulating the model, a few simplifications and constraints were assumed.

584 These will be now be reviewed and discussed in detail.

585

First of all, as stated earlier, this model has necessarily oversimplified the nature of

586

587

588

589

590

591

592

593

594

595

596

597

598

599

600 real life work collaboration issues. Real life team work is complex and dynamic. Certainly, it cannot be reduced to two variables -group diversity and social cohesion-without neglecting aspects that make from group collaboration something rich and distinct from other engineering and technical challenges. In real life, group members' exhibit behaviors and possess attributes that have not been included in this model (e.g. how introvert/extrovert group members are; their dedication, devotion, preferences or just personal or professional interests or goals; their soft skills or motivation to work in groups; the asymmetrical personal relationships as a consequence of the lines of command, etc.). However, the intention here was not to include an exhaustive list of group attributes but to present a simple and selfcontained model. And despite all the necessary simplifications, the model still seems to be robust, at least, based on the preliminary validation results shown here. The inclusion of further variables will be something that, no doubt, will be considered in future versions of the model when it is applied in real project settings.

Secondly, one might raise the question that the way group heterogeneity and cohesion have been defined in this paper might lead to some collinearity or, at least, covariance 
601 between the two constructs. This is because there is a possibility that both may be capturing some common aspects of a group configuration. Our model, however, has instrumented both

603 constructs in a multiplicative way, that is, $P_{k}^{S S} \times P_{k}^{S}$, not additive, because they do not

604 substitute for one another when contributing to subgroup performance $E_{k}$. All the same, we 605 agree that this might be an over simplification, but existing research so far does not seem to 606 have reached an agreement on whether this is an untenable assumption.

For example, Festinger et al. (1950) in an early attempt found contradicting results in two experiments analyzing the group heterogeneity-cohesion relationship. Much more recently, Dion (2000), on performing analysis in two houses of war veterans found again inconclusive findings indicating that in one house cohesion was related with homogeneity,

611 whereas in the other it was not. And even more recently, in a study conducted by Chiocchio 612 and Essiembre (2009), it was shown that a group's homogeneity or heterogeneity does not appear to affect the social cohesion-behavioral performance correlations in either academic or organizational settings. In line with this, probably the most enlightening stance has been the one taken by Sturgis et al. (2014), who claimed that the relationships between the different

616 subcomponents of group heterogeneity and cohesion might be very different from each other, 617 even cancelling out each other's effects. They also emphasized that more research is necessary to validate this. generate the most desirable subgroups allocations from the same pool of human resources,

621 the effect of potential (if existing) collinearities between subcomponents of heterogeneity and 622 cohesion will not be that critical so as to invalidate the model. This, as despite correlation

623 between both variables might cause some scale distortion, the relative rank (order) of solutions should not have altered much. 
Probably the two most relevant have been limiting the allocations of individuals to be in full-

627 time working arrangement and not part-time. Also, the relationship between group cohesion

628 and group performance has been assumed to be linear.

The first simplification is relatively easy to address but it would complicate the

630

631

632

633

634

635

636

637

638

639

640

641

642

643

644

645

646

647

648

mathematical expressions to a point where they are no longer that intuitive. In this paper, we have tried to encourage understanding of the model's utility and to avoid distractions by complicating it too much. However, allowing part-time allocations might make finding better solutions somewhat easier for an optimization algorithm. This, as the objective functions of many OR models are generally easier to optimize when the decision variables are closer to being continuous (Gutiérrez et al., 2016).

Finally, the second simplification cannot be satisfactorily addressed until there is more research to determine the nature of the cohesion-performance relationship. This might be a critical aspect for further model development. It may indeed lead to some adjustments in some of the equations (probably in expression (4) and surely in expression (14)), but for now there is no point in us speculating how it might impact the model formulation, or indeed if there is such an impact at all.

\section{Conclusions}

A model that allocates human resources to multiple projects with specific staffing requirements while also considering group homogeneity and cohesion has been proposed. This model constitutes a powerful and practical tool for any project manager who needs to efficiently allocate human resources and who wants to maximize the expected productivity of his/her group members. The mathematical expressions are, in general, quite straightforward 
649 and can be easily implemented by means of a spreadsheet. The optimization algorithm for

650 finding near-optimal solutions can also be implemented with the aid of a very simple

651 commercial solver like Excel Solver (currently a free, despite capped, version of Frontline

652 Solvers $\left.{ }^{\circledR}\right)$.

653

Human resources are a key component of project success, but there is a lack of

654

655

656

657

658

659

660

661

662

663

664

665

666

667

668

669

670

671

672

673

practical, quantitative tools that allow project managers to efficiently allocate these resources and build high performing teams. There are many reasons that can keep a team from functioning effectively. In this paper, two factors that are found to strongly and consistently influence group performance - group homogeneity and group cohesion - have been incorporated within the model. This model allows the measuring and comparing of any set of feasible subgroup allocations to several projects simultaneously.

Namely, group homogeneity has been defined by the subgroup strength metric and the sum of overlaps between subgroup members on five different demographic sub-factors (functional department, age, sex, ethnicity and team tenure). Group cohesion has been defined as the degree of acceptance (or rejection) that all members have with each other. The information on the five sub-factors in the group cohesion construct is generally very easy to obtain from the group members' professional profiles. In terms of the degree of acceptance/rejection that each group member has toward the rest of their group members, these can generally be known by using sociometric questionnaires. The latter, despite its limitations, have also been proven in previous research to be quite representative and relatively easy to use and update. Basically, these questionnaires require asking all group members who have finished a project: "Who would you like to keep working with?" and "Who would you prefer not to work with? From the group members' answers it is possible to populate (and keep updating) a sociometric matrix that is eventually useful for measuring how cohesive each potential subgroup is or can be. 

reduce/increase group performance by up to $10 \%$ on average. Similarly, group cohesion is 676 responsible for average increases (or decreases) of group performance by up to $18 \%$. Both 677 figures have been included in the proposed model and allow the objective measuring of the 678 relative group performance differences between multiple feasible subgroups. Feasible 679 subgroups are those who fulfill the minimum project staffing requirements stated by some 680 simultaneous projects.

With all this, the proposed mathematical model has been detailed concerning all its components and variable relationships. A fictitious case study involving twenty workers who are allocated to three projects have been proposed and solved by means of a simple Genetic Algorithm. Finally, a validation case study based on an academic setting has also been included which involved fifteen MSc students who were allocated to three groups and were required to complete three sequential projects. commonplace challenges of a typical project manager in efficiently allocating human resources in projects. Despite some intentional simplifications, the model shows promise in helping project managers to make more objective and efficient decisions about their human resource allocations. However, more validating studies will be required in the future to test the actual utility of the model in real project contexts. complexity of the model's application due to the number of variables to be considered, as well as generally bigger team sizes. Indeed, this wider range of variables will have some human resource implications in terms of the sturcture of social cohesion of individuals within the projects. For example, the interactions amongst members in real projects may be 
cohesion more precisely.

\section{Data availability}

All data generated or analyzed during the study are included in the submitted article

or supplemental materials files.

707

\section{References}

709

710

711

712

713

714

715

716

717

718

719

720

721

722

723

724

725

726

727

728

Agrawal, R., Golshan, B., and Terzi, E. (2014). "Grouping students in educational settings." Proceedings of the 20th ACM SIGKDD international conference on Knowledge discovery and data mining - KDD '14, ACM Press, New York, New York, USA, 10171026.

Ahmadian Fard Fini, A., Akbarnezhad, A., Rashidi, T. H., and Waller, S. T. (2017). "Job Assignment Based on Brain Demands and Human Resource Strategies." Journal of Construction Engineering and Management, 143(5), 4016123.

Ahmadian Fard Fini, A., Rashidi, T. H., Akbarnezhad, A., and Travis Waller, S. (2016). "Incorporating Multiskilling and Learning in the Optimization of Crew Composition." Journal of Construction Engineering and Management, 142(5), 4015106.

Al-Bayati, A. J., Abudayyeh, O., Fredericks, T., and Butt, S. E. (2017). "Managing Cultural Diversity at U.S. Construction Sites: Hispanic Workers' Perspectives.” Journal of Construction Engineering and Management, 143(9), 4017064.

Allison, P. D. (1978). "Measure of inequality.” American Sociological Review, 43, 865-880.

Anvuur, A. M., and Kumaraswamy, M. M. (2016). "Effects of Teamwork Climate on Cooperation in Crossfunctional Temporary Multi-Organization Workgroups." Journal of Construction Engineering and Management, 142(1), 4015054.

Awal, G. K., and Bharadwaj, K. K. (2014). "Team formation in social networks based on collective intelligence - an evolutionary approach." Applied Intelligence, Kluwer Academic Publishers, 41(2), 627-648.

Ballesteros-Pérez, P., González-Cruz, M. C., and Fernández-Diego, M. (2012). "Human resource allocation management in multiple projects using sociometric techniques." International Journal of Project Management, 30(8).

Beal, D. J., Cohen, R. R., Burke, M. J., and McLendon, C. L. (2003). "Cohesion and 
performance in groups: a meta-analytic clarification of construct relations." The Journal of applied psychology, 88(6), 989-1004.

Blau, P. M. (1977). Inequality and Heterogeneity. Free press, New York.

Castaño, N., Watts, T., and Tekleab, A. G. (2013). "A reexamination of the cohesionperformance relationship meta-analyses: A comprehensive approach." Group Dynamics: Theory, Research and Practice, 17(4), 207-231.

Chang, A., and Bordia, P. (2001). "A Multidimensional approach to the Group Cohesion Group Performance relationship.” Small Group Research, 32(4), 379-405.

Chen, S., Wang, D., Zhou, Y., Chen, Z., and Wu, D. (2017a). "When too little or too much hurts: Evidence for a curvilinear relationship between team faultlines and performance." Asia Pacific Journal of Management, Asia Pacific Journal of Management, 34(4), 931950.

Chen, Y., McCabe, B., and Hyatt, D. (2017b). "Relationship between Individual Resilience, Interpersonal Conflicts at Work, and Safety Outcomes of Construction Workers." Journal of Construction Engineering and Management, 143(8), 4017042.

Chiocchio, F., and Essiembre, H. (2009). Cohesion and Performance: A Meta-Analytic Review of Disparities Between Project Teams, Production Teams, and Service Teams. Small Group Research.

Dion, K. L. (2000). “Group cohesion: From 'Field of Forces' to Multidimensional Construct." Group Dynamics: Theory, Research, and Practice, 4(1), 7-26.

Dorn, C., and Dustdar, S. (2010). On the Move to Meaningful Internet Systems: OTM 2010. Lecture Notes in Computer Science (including subseries Lecture Notes in Artificial Intelligence and Lecture Notes in Bioinformatics), Lecture Notes in Computer Science, (R. Meersman, T. Dillon, and P. Herrero, eds.), Springer Berlin Heidelberg, Berlin, Heidelberg.

Earley, P. C., and Gibson, C. B. (2002). Multinational Work Teams: A New Perspective. Mahwah, N.J.: Lawrence Erlbaum Associates.

Evans, C. R., and Dion, K. L. (2012). "Group Cohesion and Performance: A Meta-Analysis." Small Group Research, 43(6), 690-701.

Farhadi, F., Hoseini, E., Hashemi, S., and Hamzeh, A. (2012a). "TeamFinder: A Coclustering based Framework for Finding an Effective Team of Experts in Social Networks." 2012 IEEE 12th International Conference on Data Mining Workshops, IEEE, 107-114.

Farhadi, F., Sorkhi, M., Hashemi, S., and Hamzeh, A. (2011). "An Effective Expert Team Formation in Social Networks Based on Skill Grading." 2011 IEEE 11th International Conference on Data Mining Workshops, IEEE, 366-372.

Farhadi, F., Sorkhi, M., Hashemi, S., and Hamzeh, A. (2012b). "An Effective Framework for Fast Expert Mining in Collaboration Networks: A Group-Oriented and Cost-Based Method." Journal of Computer Science and Technology, 27(3), 577-590.

Festinger, L., Schachter, S., and Back, K. (1950). Social pressures in informal groups; a study of human factors in housing. Harper, Oxford, England.

Gajewar, A., and Sarma, A. Das. (2012). "Multi-skill collaborative teams based on densest subgraphs." Proceedings of the 12th SIAM International Conference on Data Mining, SDM 2012, 165-176. 
Gibson, C., and Vermeulen, F. (2003). "A Healthy Divide : Subgroups as a Stimulus for Team Learning Behavior.” Administrative Science Quarterly, 48(2), 202-239.

Gutiérrez, J. H., Astudillo, C. A., Ballesteros-Pérez, P., Mora-Melià, D., and Candia-Véjar, A. (2016a). "The multiple team formation problem using sociometry." Computers and Operations Research, 75.

Gutiérrez, J. H., Astudillo, C. A., Ballesteros-Pérez, P., Mora-Melià, D., and Candia-Véjar, A. (2016b). "The multiple team formation problem using sociometry." Computers and Operations Research, Elsevier, 75, 150-162.

Hendriks, M. H. A., Voeten, B., and Kroep, L. (1999). "Human resource allocation in a multi-project R\&D environment: Resource capacity allocation and project portfolio planning in practice." 17(3), 181-188.

Lappas, T., Liu, K., and Terzi, E. (2009). "Finding a team of experts in social networks." Proceedings of the 15th ACM SIGKDD international conference on Knowledge discovery and data mining - KDD ’09, ACM Press, New York, New York, USA, 467.

Lau, D. C., and Murnighan, J. K. (2005). "Interactions within Groups and Subgroups: The Effects of Demographic Faultlines." The Academy of Management Journal, Academy of Management.

Li, C.-T., and Shan, M.-K. (2010). "Team Formation for Generalized Tasks in Expertise Social Networks." 2010 IEEE Second International Conference on Social Computing, IEEE, 9-16.

Lott, A. J., and Lott, B. E. (1965). "Group cohesiveness as interpersonal attraction: A review of relationships with antecedent and consequent variables." Psychological Bulletin, 64(4), 259-309.

Mathieu, J. E., Kukenberger, M. R., D’Innocenzo, L., and Reilly, G. (2015). “Modeling Reciprocal Team Cohesion - Performance Relationships , as Impacted by Shared Leadership and Members ' Competence Modeling Reciprocal Team Cohesion Performance Relationships , as Impacted by Shared Leadership and Me." Journal of Applied Psychology, 100(3), 713-734.

Meyer, B., and Glenz, A. (2013). "Team Faultline Measures: A Computational Comparison and a New Approach to Multiple Subgroups." Organizational Research Methods, 16(3), $393-424$.

Moreno, J. L. (1941). "Foundations of Sociometry: An Introduction.” Sociometry, 4(1), 1535.

Phua, F. T. T. (2004). "The antecedents of co-operative behaviour among project team members: an alternative perspective on an old issue." Construction Management and Economics, Taylor \& Francis Group , 22(10), 1033-1045.

Phua, F. T. T., and Rowlinson, S. (2004). "How important is cooperation to construction project success? A grounded empirical quantification." Engineering, Construction and Architectural Management, Emerald Group Publishing Limited, 11(1), 45-54.

Salo, M. (2006). The Relation Between Sociometric Choices and Group Cohesion. Arlington, Virginia.

Shi, Z., and Hao, F. (2013). "A strategy of multi-criteria decision-making task ranking in social-networks." The Journal of Supercomputing, 66(1), 556-571.

Sorkhi, M., Alvari, H., Hashemi, S., and Hamzeh, A. (2012). “A game-theoretic framework 
to identify top-k teams in social networks." KDIR 2012 - Proceedings of the

Sturgis, P., Brunton-Smith, I., Kuha, J., and Jackson, J. (2014). "Ethnic diversity, segregation and the social cohesion of neighbourhoods in London." Ethnic and Racial Studies, Taylor \& Francis, 37(8), 1286-1309.

Teixeira, L. O., and Huzita, E. H. M. (2014). DiSEN-AlocaHR: A Multi-Agent Mechanism for Human Resources Allocation in a Distributed Software Development Environment International Conference. Advances in Intelligent Systems and Computing, (S. Omatu, H. Bersini, J. M. Corchado, S. Rodríguez, P. Pawlewski, and E. Bucciarelli, eds.), Springer International Publishing, Cham.

Tseng, T.-L. (Bill), Huang, C.-C., Chu, H.-W., and Gung, R. R. (2004). "Novel approach to multi-functional project team formation." International Journal of Project Management, 22(2), 147-159.

Xia, N., Zhong, R., Wu, C., Wang, X., and Wang, S. (2017). “Assessment of StakeholderRelated Risks in Construction Projects: Integrated Analyses of Risk Attributes and Stakeholder Influences." Journal of Construction Engineering and Management, 143(8), 4017030.

Yin, H., Cui, B., and Huang, Y. (2011). Advanced Data Mining and Applications. Lecture Notes in Computer Science (including subseries Lecture Notes in Artificial Intelligence and Lecture Notes in Bioinformatics), Lecture Notes in Computer Science, (J. Tang, I. 